the type of results obtained. These particular results, given as live units, were secured under midsummer conditions in 1929 ; the bait, half a sheep's head laid on a piece of mutton, contained approximately $1 \mathrm{kgm}$. of consumable meat. The initial population of $L$. sericata was approximately 50,000 (the decreasing population twenty-four hours after exposure was 48,562 ), while the final field emergence was 231 flies. During the summer of 1930 , under slightly hotter conditions, with a rabbit bait of approximately equal weight of consumable meat, and with Chrysomyia playing a role comparable to that of Sarcophagids in the former bait, an initial population of approximately $60,350 \mathrm{~L}$. sericata plus $2850 \mathrm{~L}$. cassar yielded a final Lucilia population of 30 while the final Chrysomyia population was 2611 .

Faculté des Sciences,

Toulouse, France.

\section{Determination of the Abundance Ratios of Isotopes from Band Spectra.}

IN band-spectroscopic determinations of the abundance ratio of isotopes, an important but hitherto neglected point of view appears as soon as one attempts to make any calculations of greater accuracy.

Suppose the isotopes $A_{1}$ and $A_{2}$ exist in the abundance ratio $m$. In molecular compounds with atoms $B$ they will form molecules $A_{1} B$ and $A_{2} B$ also in the abundance ratio $m$. Now raising the temperature of the mixture, so that several vibrational states of the molecules come into play, this abundance ratio will not remain fixed for each state. Provided there is a thermodynamical equilibrium, the molecules will be distributed according to the Boltzmann expression $A e^{-E / k T}$, where $E$, the vibrational energy of the molecules, is different for different isotopes.

Carryirg out such calculations on boron monoxide, $\mathrm{B}^{11} \mathrm{O}$ and $\mathrm{B}^{10} \mathrm{O}$, at room temperature, the abundance ratio of their normal state $(v=0)$ is found to be approximately equal to $m$. But already in the first excited vibrational state $(v=1)$ this ratio is changed into $1.3 m$. From the Condon parabola of the intensity distribution in the $\beta$-bands of boron monoxide one finds by the aid of the Franck.Condon principle that transitions from the normal state $(v=0)$ chiefly will hit the first three vibrational levels $\left(v^{\prime}=0,1,2\right)$ in the excited electronic state. A. Elliott (NATURE, 126, 203; 1930) photographed this spectrum, using activated nitrogen as the source of emission. From intensity measurements on different plates he finds an approximately constant abundance ratio $m$ for bands corresponding to transitions from $v^{\prime}=1,2$ in the excited state. However, in bands corresponding to transitions from $v^{\prime}=3$ he finds a remarkable increase of 30 per cent in this value. This observation is in perfect agreement with our calculation above, as the population of molecules in the vibrational state $v^{\prime}=3$ is chiefly fed through collisions between active nitrogen and the boron monoxide molecules from $v=1$ in the normal state. Moreover, the results of Elliott indicate that the Franck-Condon principle is valid also in this special case of low temperature, where the electronic transition is caused through collisions between particles of approximately equal masses.

Occasionally, Naudé's results on the determination of the abundance ratio of $\mathrm{O}^{16}$ and $\mathrm{O}^{18}$ (Phys. Rev., 36, 333 ; 1930) may be correct as based on intensity measurements of bands corresponding to transitions from the normal state $v=0$ in the $N O(\beta)$ spectrum.

Generally, however, correct values of the abundance ratio $m$ from band-spectroscopic data afford de- terminations of $m$ as a function of $v$, taking their weighted mean values from the energy distribution in the gas. Practically correct values are also obtained through intensity measurements on absorption bands belonging to $v=0$ in the normal state, if the absorption gas is kept at low temperature.

Laboratory of Physics, G. Stenvinket.

University of Stockholm Sept. 12.

\section{Change of the Dielectric Constant of Ethyl Ether with Temperature.}

I HAVE made a study of the dielectric constant of ethyl other as a function of temperature with the aid of high frequency electromagnetic oscillations, using the method described by M. Wolfke and W. Keesom, Comm. Leiden, $190 a$.

The construction of the measuring condenser has permitted the cooling of ethyl ether down to $-150^{\circ} \mathrm{C}$. by the use of petrol ether as a cooling liquid, which was contained in a Dewar vessel, provided with a special refrigerator, cooled with liquid air. The uniformity of temperature throughout the substance under investigation was ensured by the use of a double stirrer and by the thinness of the layer of the cooling liquid. Temperatures were determined by means of

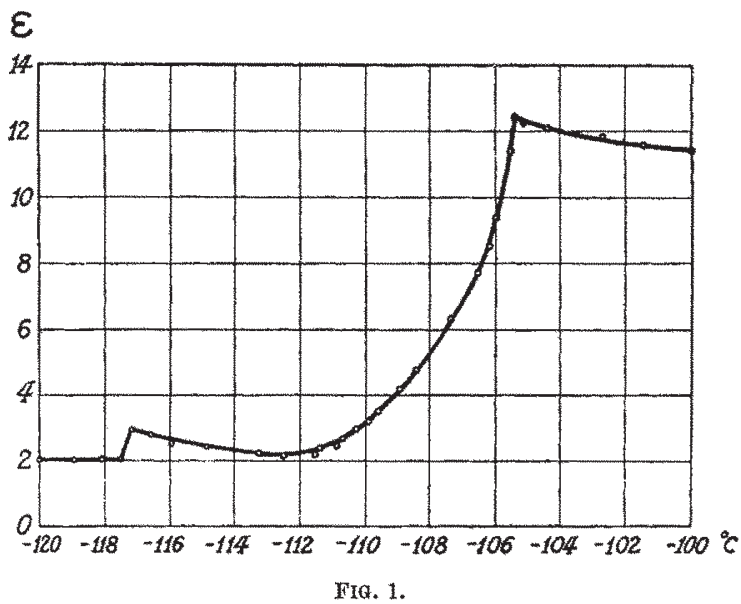

a platinum resistance thermometer, wound on the surface of the measuring condenser and calibrated with the aid of the normal thermometer of the Cryogenic Laboratory at Leyden.

The dielectric constant of very carefully purified ethyl ether increases with the lowering of temperature from $4 \cdot 18$ at $30 \cdot 6^{\circ} \mathrm{C}$. up to the highest value $12 \cdot 39$ at $-105 \cdot 4^{\circ} \mathrm{C}$. and decreases very rapidly beyond that point. At the melting point, $-117 \cdot 2^{\circ} \mathrm{C}$., there appears a distinct change in the dielectric constant, which was not observed by any of the previous workers. At temperatures lower than $-118.9^{\circ}$ the dielectric constant has a nearly constant value, equal to $2 \cdot 04$.

The changes of the dielectric constant of ethyl ether with the temperature described above are represented on the accompanying graph (Fig. 1).

Physical Laboratory,

J. Mazur.

Technical Institute, Warsaw, Sept. 30. 